\title{
THE POPULATION I CONTENT OF THE ELLIPTICAL COMPANIONS OF M31
}

\author{
P. W. HODGE \\ University of Washington, Seattle, Wash., U.S.A.
}

(To be published elsewhere)

\begin{abstract}
Twenty years ago Walter Baade pointed out the remarkable fact that two of the four elliptical galaxy companions of M31 (namely NGC 185 and 205) contained apparent Population I in their central areas, evidenced by the presence of dust clouds and a dozen or so OB stars. I wish to report here on some observations of these stars and absorption regions, made in an attempt to understand the origin of this anomalous material and its relevance, if any, to the pattern of galaxy development.
\end{abstract}

\section{Methods of Observation}

Direct photoelectric measures with small diaphragms were made every few seconds along the NS and EW axes of the galaxies, all in the U, B, V system. Schmidt plates taken with the 48-inch Palomar instrument were used to produce isophotes over a wide range of distances from the galaxy centers. For the OB stars, Arp's sequence in M31 was used with astrophotometer measures to establish local standards near NGC 205, and then Baade's 200-in. plates were used to transfer magnitudes therefrom to the embedded OB stars.

\section{Color Distribution}

The color distributions of NGC 185 and 205 are quite similar. Both show a blue excess at the center that decreases outward to a limit, and the color then remains constant further outward. The data are:

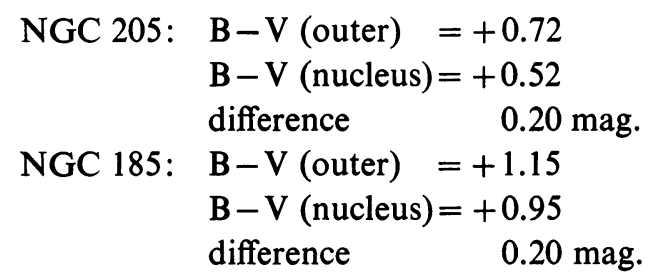

The color anomaly in NGC 205 is noticeably offset from the center in the same sense as the $\mathrm{OB}$ stars.

\section{The OB Stars}

The best of Baade's 200-in. telescope plates show as many as 61 OB stars in NGC 205 and nearly as many in NGC 185. In NGC 205 the centroid of these stars is offset from the nucleus by $30 \mathrm{pc}$ west and $33 \mathrm{pc}$ north. For NGC 185 they are nearly centered. The sizes of the Population I stellar arrays are 335 pc for NGC 205 and approximately 270 pc for NGC 185. The stars in NGC 205 are arranged in an irregular elliptical 
grouping with its major axis lined up with that of the galaxy's old stars. The brightest star in NGC 205 has $M_{B}=-5.2$, implying an age of $4 \times 10^{6} \mathrm{yr}$, if at the main sequence turn-off. Masses for the Population I components for the two galaxies are $5 \times 10^{5} \mathscr{M}_{\odot}$ for NGC 205, based on the available luminosity function assumed to extend parallel to the van Rhijn function to $M_{V}=+3.5$, and $2 \times 10^{5} \mathscr{M}_{\odot}$ for NGC 185 , based on the total color and a similar assumption about the luminosity function.

\section{The Dust Lanes}

NGC 205 has two large dust lanes and more than a dozen small, faint ones. For the largest the measured color excess is $\Delta(\mathrm{B}-\mathrm{V})=+0.13$ implying total absorption of $\Delta \mathrm{V}=0.4 \mathrm{mag}$., using the usual assumptions. For the two absorption regions in NGC 185 the total absorption measures average 0.3 and 0.15 , and total masses are estimated to be 200 and $25 \mathscr{M}_{\odot}$, respectively.

\section{Discussion}

Heidmann: Have you any comment on the fact that in NGC 205 the dust is on one side, and the O and $B$ stars are on the other side?

Hodge: The dust clouds are clustered in an area southwest of the NGC 205 nucleus at a distance almost identical to that of the OB star centroid, in the opposite direction. It is conceivable that this causes obscuration of the fainter stars and that the asymmetry in the OB star distribution is only apparent, the result of the real asymmetry in dust. Alternatively, the southwest part of the Population I material, if it is truly symmetrical in mass, may be relatively less condensed into stars there, in which case perhaps neutral hydrogen should be searched for near the dust regions. The total dust mass cannot be more than a percent or so of the computed mass contributed by the Population I stars. 